\title{
What are mechanisms of re-sedation caused by remimazolam?
}

\author{
Kohei Godai ${ }^{1}$ (]) \\ Received: 11 March 2021 / Accepted: 16 March 2021 / Published online: 22 March 2021 \\ (c) Japanese Society of Anesthesiologists 2021
}

To the Editor:

I read with great interest the article "Re-sleeping after reversal of remimazolam by flumazenil" by Yamamoto et al. [1]. I think that this report is important, because rare phenomena only appear after a large amount of the new drug is used. I believe, however, that several issues need to be discussed. First, what are mechanisms of re-sedation caused by remimazolam? The authors provide little information about the case. Remimazolam package insert says "half-life is prolonged with increasing severity of hepatic impairment". I think that the following information would be useful for the readers, perioperative opioids usage, patient's past history including liver/kidney functions, sedation score and respiratory rate when the patient was under re-sedation. Second, the authors used flumazenil $0.5 \mathrm{mg}$ immediately after remimazolam cessation. Why did the authors select the timing and dosage of flumazenil? The authors state that "all recommended dosages are used". There is, however, no recommendation dosage about flumazenil [2]. Third, the authors seem to be confused about drug half-life. CSHT is defined as "the time required for the plasma drug concentration to decline by $50 \%$ after terminating each infusion" [3]. Although elimination half-life of remimazolam is about $40 \mathrm{~min}$, CSHT is less than $10 \min [4]$.
Acknowledgements None.

Author contributions $\mathrm{KG}$ wrote the manuscript.

\section{Declarations}

Conflict of interest None.

\section{References}

1. Yamamoto T, Kurabe M, Kamiya Y. Re-sleeping after reversal of remimazolam by flumazenil. J Anesth. 2021;35:322.

2. Hirata N, Hayamizu K, Yamakage M. How to administer remimazolam for anesthesia induction. J Anesth. 2020;34:962.

3. Hughes MA, Glass PS, Jacobs JR. Context-sensitive half-time in multicompartment pharmacokinetic models for intravenous anesthetic drugs. Anesthesiology. 1992;76:334-41.

4. Wesolowski AM, Zaccagnino MP, Malapero RJ, Kaye AD, Urman RD. Remimazolam: pharmacologic considerations and clinical role in anesthesiology. Pharmacotherapy. 2016;36:1021-7.

Publisher's Note Springer Nature remains neutral with regard to jurisdictional claims in published maps and institutional affiliations.

Kohei Godai

kxg179@icloud.com

1 Department of Anesthesiology and Critical Care

Medicine, Graduate School of Medical and Dental

Sciences, Kagoshima University, 8-35-1 Sakuragaoka,

Kagoshima 890-8520, Japan 